\title{
A light touch
}

\author{
Since the discovery of the optical gradient force in 1970 and the first use of laser beams to manipulate \\ microscopic and atomic systems in 1986, optical manipulation has proved to be a versatile optical tool \\ for uncovering mysteries throughout many fields of science.
}

An optical trap is based on the forces derived from the radiation pressure exhibited by a high-power laser beam. When laser light is tightly focused to a diffraction-limited spot, a steep three-dimensional gradient of light can be formed in the vicinity of the focus. Combined with additional forces due to light scattering and gravity, the resulting gradient creates a stable trap position close to the focal point of the beam that draws dielectric (transparent) particles towards the focus. This simple working principle allows an optical trap to capture and stably manipulate micro- and nanoscale particles, thus facilitating the experimental study of small particles in many fields.

This issue of Nature Photonics has a special focus on optical manipulation and trapping, featuring an Interview with Arthur Ashkin, the pioneer of optical tweezers, as well as three Reviews and two Commentaries that discuss the different approaches for trapping and controlling particles and summarize the applications of optical manipulation in the biological and physical sciences.

In the Interview on page 316, Arthur Ashkin recalls his discovery of the optical gradient and scattering forces around four decades ago. He recalls that there were many exciting moments during his research, the most invigorating being the realization that light could be used to trap atoms and molecules. He persevered despite discouragement from bosses, and his belief in the potential of the optical tweezer trap led not only to his success but also the success of many other researchers in the field. Ashkin explains that optical tweezers have significant potential in fields such as quantum computing, disease diagnosis, optical cooling, superconductivity, gravitational detection and the precise measurement of fundamental constants.

Another notable achievement of optical tweezers is their application to biological studies at the single-molecule level. Indeed, the ability of optical tweezers to access nanometre-length distances and piconewton forces makes them one of the most important tools for a biophysicist. On page 318, Furqan Fazal and Steven Block from Stanford University in the USA explain that

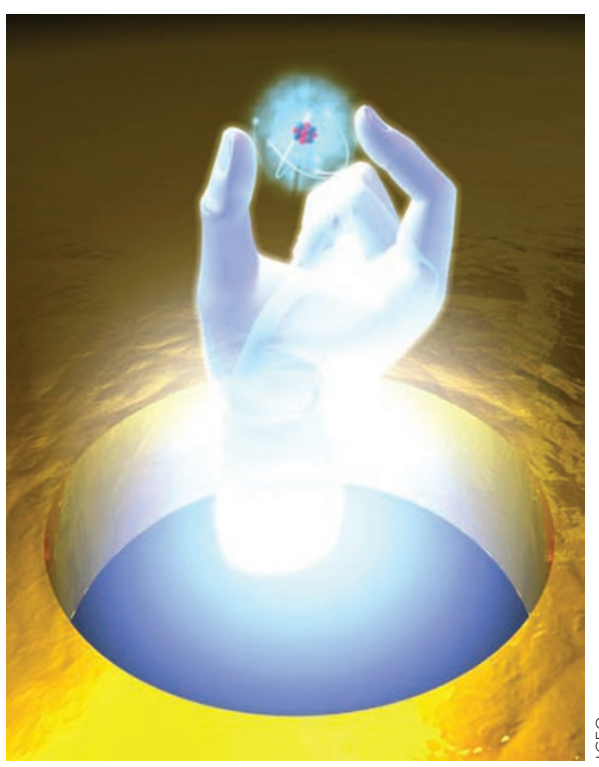

that often destructs a stable trap. On page 343, Miles Padgett and Richard Bowman from the University of Glasgow in the UK explain how light beams carrying orbital angular momentum (demonstrated almost two decades ago in a Laguerre-Gaussian mode) can be used to solve this problem, and how their unique intensity, polarization and phase structure properties are well-suited to optical manipulation.

Optical manipulation can also be achieved by sculpting the shape of an object, as recently demonstrated by Grover Swartzlander and co-workers from Rochester University of Technology in the USA ${ }^{1}$ - a finding that has added a new and revitalizing twist to the field of optical manipulation.

The latest advances in nano-optics, specifically in the field of plasmonics, now allow low-intensity evanescent optical fields to achieve in-plane, parallel and selective manipulation of nanoparticles. On page

optical tweezers function like microscale three-dimensional springs that can directly grasp, manipulate and exert controlled forces. Such forces can be translational or rotational, in a single trap or multiple traps, and act on either single molecules or molecular systems comprised of several biomolecules. Optical tweezers have made many contributions to our understanding of the mechanochemistry of molecular motors and the nanomechanical properties of biological polymers, and will continue to flourish in the field of single-molecule biophysics, according to Fazal and Block.

Although most optical trapping schemes exploit standard Gaussian beams, shaping the phase and amplitude of the optical field can be used to create unusual light patterns that enable multiparticle trapping. On page 335, Kishan Dholakia and Tomas Čižmár from the University of St Andrews in the UK describe how light can be shaped using diffractive optics and holography, and how shaped 'non-diffracting' fields - particularly Bessel, Airy and Mathieu beams - have led to new discoveries in microscopic science. They also describe how optical lattices and disorders can be exploited for optical manipulation.

Although optical tweezers work well for most dielectric particles, absorbing particles experience a much higher scattering force 349, Mathieu Juan, Maurizio Righini and Romain Quidant from the Institut de Ciències Fotòniques and the Institució Catalana de Recerca i Estudis Avançats in Spain review the recent advances of plasmon-based optical trapping and discuss the potential applications of plasmonic tweezers to the fields of bioscience and quantum optics.

Another emerging technique is the use of optoelectronic tweezers - a manipulation approach that exploits both optical and electrical bias. On page 322, Ming Wu from the University of California at Berkeley in the USA explains how these devices create virtual electrodes by projecting light patterns onto a photosensitive substrate. The resulting non-uniform electric field then exerts forces on dielectric particles through dielectrophoresis. Wu points out that the main advantage of this technique is that both dynamic optical addressability and large forces can be achieved at low light intensities, thus minimizing damage to the sample.

The continued development and growing sophistication of optical manipulation techniques will undoubtedly facilitate new and exciting discoveries for many years to come.

\section{References}

1. Swartzlander, G. A. Jr, Peterson, T. J., Artusio-Glimpse, A. B. \& Raisanen, A. D. Nature Photon. 5, 48-51 (2011) 